\title{
Robust Control of a Deformable Mirror for Adaptive Optics Systems
}

\author{
Dingan Song ${ }^{*}, 1,2,3,4$ Xinyang $\mathrm{Li}^{*}, 1,3$ and Zhenming Peng ${ }^{2}$ \\ ${ }^{1}$ Key Laboratory on Adaptive Optics, Chinese Academy of Sciences, Chengdu, Sichuan, China; \\ ${ }^{2}$ University of Electronic Science and Technology of China, Chengdu, Sichuan, China; \\ ${ }^{3}$ Institute of Optics and Electronics, Chinese Academy of Sciences, Chengdu, Sichuan, China; \\ ${ }^{4}$ University of Chinese Academy of Sciences, Beijing, China \\ ${ }^{*}$ Corresponding author
}

\begin{abstract}
To improve the robust stability of adaptive optics system, $H_{\infty}$ synthesis has been introduced to design the controller. $H_{\infty}$ synthesis has advantages to deal with unmodelled dynamic of plant and to ensure the optimal performance in tracking the desired input. To simplify the design of controller, Singular Value Decomposition is adopted to uncouple the channels. This paper first analyzes the frequency responses of the $H_{\infty}$ synthesis and integrator in uncoupled channels. The results indicate that $H_{\infty}$ synthesis can increase the phase margin, which explain that the stability of adaptive optics is improved. Then the simulation of atmospheric turbulence correction is analyzed to testify the performance of adaptive optics. The results show that both integrator and $H_{\infty}$ synthesis can obtain a nearly same satisfying performance, but the former achieves a lower control voltage to correct wavefront than the latter. It validates that $H_{\infty}$ synthesis has a better robustness to deal with the disturbance than integrator. That is to say, $H_{\infty}$ synthesis is able to improve the stability of adaptive optics system.
\end{abstract}

Keywords- $H_{\infty}$ synthesis; adaptive optics; atmospheric correction

\section{INTRODUCTION}

Adaptive Optics (AO) is a mature technology that has been successfully implemented to reduce the degrading effects of the Earth's atmosphere on optical astronomical observations. Almost all modern large telescopes are equipped with AO systems to improve image resolution. As the performance of $\mathrm{AO}$ systems (which can effectively correct the atmospheric turbulence) is decided by controller, the design of controller becomes increasingly important. Up to now, the classic integrator controller is the default method in AO engineering. This method measures the residual wavefront (usually results from the Shack-Hartmann detector) from the AO system and modifies the parameters easily to achieve a good performance. However, due to simple structure and limited parameter of integrator controller, AO system equipped with integrator cannot obtain an excellent performance.

To improve the performance of AO system, a variety of modern control strategies have been introduced to design the controller. For examples, References [1-4] introduce robust control to improve the robust stable of AO system and seek for the optimal correction ability. References [5-8] use LQG strategy to minimize the variance of the residuals and improve AO system performance under several conditions. References [9-12] use adaptive control strategy to deal with the change of the turbulence. And references [13-16] apply the predicting control to deal with the time delay of the AO system. While these advanced control algorithms could enhance the performance of AO system, they are hard to be applied to engineering for reasons as the following reasons:

i) Modern control depends on the mathematic model of the plant heavily and the precise model is hard to be established.

ii) Controller designed based on modern control theory is usually too complex to be applied to engineering.

iii) It is difficult for modern control to suit all states in engineering, because the states of plant may change in actual environment.

Despite many problems, modern control makes it possible to improve the performance of $\mathrm{AO}$ system. Its feasibility drives researchers to study modern control in AO system. This article researches the $H_{\infty}$ synthesis based on the following reasons:

- $H_{\infty}$ Synthesis excels at dealing with unmodelled dynamics and unknown disturbance.

- $H_{\infty}$ Synthesis aims at flattening a mixed norm of residuals and noise. It is amendable to meaningful optimization and can deal with multivariable problem.

- Though the mathematic theory of $H_{\infty}$ synthesis is very difficult, the realization of $H_{\infty}$ controller is simple and easy.

- It is easy for $H_{\infty}$ synthesis to achieve excellent performance by choosing appropriate weight functions. For instance, A. Guesalaga et al added a notch on the weight function of sensitivity function to mitigate vibration at specific frequency [17-20].

Our approach aims at improving the stability while guaranteeing the correction ability of AO system. Two control laws have been implemented: the classic integrator 
and $H_{\infty}$ synthesis. In [21] we have presented preliminary results of $H_{\infty}$ synthesis for single-input and single-output (SISO) loop. However, AO system is a multi-input and multi-output (MIMO) plant. This paper analyzes the frequency response and simulates in the closed loop with two layer turbulence simulative date.

The structure of the paper is as follows: Section 2 introduces the plant model and its decomposition. Section 3 explains the theory behind the controller implemented here. Section 4 conducts frequency analysis of the system. Section 5 presents the results from numerical simulation for artificially generated turbulence. Finally in section 6, conclusions are presented.

\section{PlANT MODEL AND DECOMPOSITION}

A typical AO feedback control system shown in Figure. I consists of three primary components: a wavefront sensor (WFS) to detect the phase aberration, a deformable mirror (DM) to correct the detected aberration and a control computer (Controller) to calculate the command for the DM. A beam with a known phase called reference beam is usually used as a reference against which the aberration introduced in the optical path is measured. Shack-Hartmann (SH) WFS is one of the common wavefront sensors used in AO systems. It measures the local gradients of the phase by an array of lenslets which produces a grid pattern of bright spots on a CCD or CMOS camera located at the focal length of the lenslets. The controller sends control signal calculated from the error signal (detected by the $\mathrm{SH}$ ) to command the DM to work.

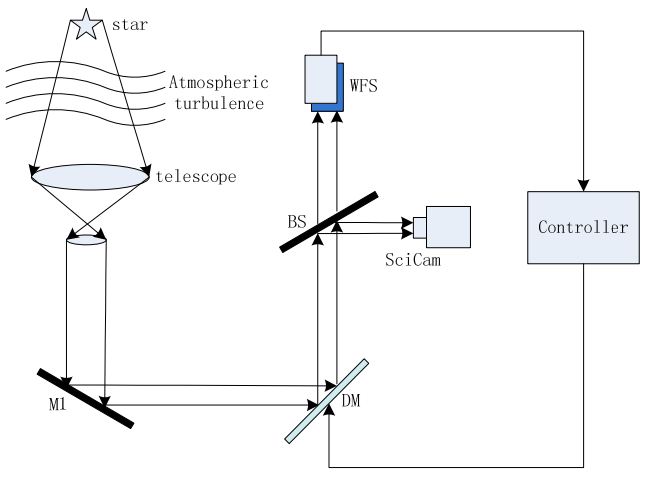

FIGURE I. TYPICAL ADAPTIVE OPTICS SYSTEMS WITH SHACK HARTMANN WAVEFRONT SENSOR

\section{A. Plant Model}

The typical AO feedback control system can be represented by an equivalent diagram from control point of view as shown in Figure. II, and the distribution of actuators and subapertures over the DM unitary surface is shown in Figure. III, where dots denote the location of actuators and squares denote the subapertures. The actuator command is represented by vector $u$ and the sensor output is represented by vector $\varphi_{y}$. These vectors form two vector spaces are called control space and sensor space. The dimension of the control space and the sensor space are the number of the actuator channels and twice the number of the sensor channels (for SH WFS, the output is the $\mathrm{x}$ and $\mathrm{y}$ component of the phase gradient), respectively. The disturbance is represented by a matrix $\varphi_{d}$ and residual wavefront is represented by a matrix $\varphi_{e}$. Both the two matrixes are defined in the sensor space. Vector $n$ represents noises which include the camera read out noise and unmodelled dynamic of plant etc. The error signal is denoted by vector $e . K(s)$ denotes the transfer function of controller, and $P(s)$ denotes the mathematic model of the plant.

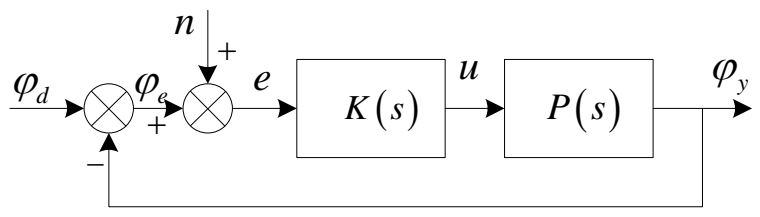

FIGURE II. DIAGRAM OF ADAPTIVE OPTICS SYSTEM FROM CONTROL PERSPECTIVE

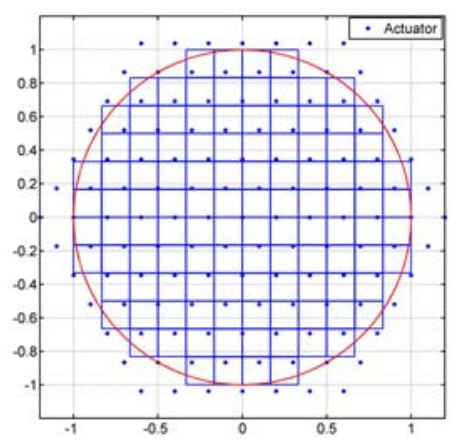

FIGURE III. LOCATION OF ACTUATORS AND SHACK-HARTMANN SUBAPERTURES OVER THE DM UNITARY SURFACE; DOT: ACTUATORS (127) AND SQUARE: SUBAPERTURES (112).

The path from $u$ to $\varphi_{y}$ is a multi-input and multi-output (MIMO) plant which can be represented by the following continuous-time transfer function model.

$$
[P(s)]_{2 b \times a}=\frac{\varphi_{y}(s)}{u(s)}=\left[\begin{array}{ccccc}
p_{11}(s) & & \ldots & & p_{1 a}(s) \\
& \ddots & & & \\
\vdots & & p_{i j}(s) & & \vdots \\
& & & \ddots & \\
p_{2 b \times 1}(s) & & \ldots & & p_{2 b \times a}(s)
\end{array}\right](1)
$$

The scalar transfer function $p_{i j}(s)$ in $P(s)$ depends on the frequency response of the plant (from $i$-th actuator to $j$-th subapertures), subscript $b$ is the number of subapertures, and the subscript $a$ is the number of actuator. Since the responsive bandwidth of DM is high enough, its dynamics can be ignored. The plant is often approximated by a constant matrix $D$ (called influence matrix or transfer matrix) with a time delay operator separated from the dynamics. The influence matrix is the steady state response 
of the plant to a unit step input. Then $p_{i j}(s)$ can be written as

$$
p_{i j}(s)=d_{i j} e^{-\tau_{i j} s}
$$

The value of $d_{i j}$ is obtained from the influence matrix, and can be united in the uncouple loop. Delay operator $\tau_{i j}$ is the time delay from the $j$-th actuator input to $i$-th output of detector, which includes camera exposure time, read out time, and computer calculate time and high voltage holding time. For line transfer operating mode, the signals are read out line by line, so the time delay is different from some loop. Usually, it is about 2-3 sampling cycles. The influence matrix $D$ can be calculated from the next equation [22]. The slope response from $j$-th actuator to $i$-th subapertures is

$$
\left\{\begin{array}{l}
d_{i j x}=\frac{1}{s_{j}} \iint_{s_{j}} \frac{\partial R_{i}(x, y)}{\partial x} d x d y \\
d_{i j y}=\frac{1}{s_{j}} \iint_{s_{j}} \frac{\partial R_{i}(x, y)}{\partial y} d x d y
\end{array}\right.
$$

Where $s_{j}$ is the unitary area of the $j$-th subapertures and $R_{i}(x, y)$ is the response function of most zonal deformable mirrors using continuous faceplates. The subscript $\mathrm{x}$ and $\mathrm{y}$ represent $\mathrm{x}$ direction and $\mathrm{y}$ direction respectively. The response function of $i$-th actuator $R_{i}(x, y)$ can be approximated by a super-Gaussian function as follow [23]:

$$
R_{i}(x, y)=\exp \left[\left(\ln a_{m}\right)\left(\frac{\sqrt{\left(x-x_{i}\right)^{2}+\left(y-y_{i}\right)^{2}}}{r_{m}}\right)^{\alpha}\right]
$$

\section{Where}

$$
\begin{aligned}
& x, y=\text { coordinates in the plane of the mirror; } \\
& a_{m} \quad=\text { a constant defining the actuator sensitivity; } \\
& x_{i}, y_{i}=\text { specification of the } i \text {-th actuator locations; } \\
& \alpha \quad=\text { Gaussian index; } \\
& r_{m} \quad=\text { space between adjacent actuators. }
\end{aligned}
$$

So the influence matrix can be obtained by calculating or measuring.

\section{B. Model Decomposition}

For AO system involving larger flexible structures, the design of controller in direct way is too difficult to be applied to engineering. To simplify the design, this paper uncouples the input and output channels of the plant before designing the controller. Once uncoupled, an SISO controller can be applied to the uncoupled channels.
Singular Value Decomposition (SVD) is a widely used method of model decomposition. It offers two advantages [24]: one is that the SVD basis is orthogonal and can guarantee the stability of the solution of controller. The other is that the basis vectors are ordered by the magnitudes of the associated singular values which can be regarded as the gain of the plant.

The SVD of influence matrix $D$ is defined as

$$
D=U \Sigma V^{T}
$$

Where $U$ and $V$ are matrices whose column vectors are orthogonal and normalized. For the size of influence matrix $D$ is $2 b \times a$, the size of $U$ and $V$ are $2 b \times 2 b$ and $a \times a$, respectively. The matrix $\Sigma$ is a $2 b \times a$ diagonal matrix whose diagonal components are the singular values of the influence matrix $D$. Typically $2 b \neq a$ and the rank of $D$ is $r=\min \{a, 2 b\}$, only the first $r$ diagonal components of $\Sigma$ have non-zero values and $\Sigma$ can be reduced to an $r \times r$ matrix with $U$ and $V$ correspondingly reduced to have only the first $r$ columns. That is called thin SVD and can be represented as follows.

$$
D=U_{r} \Sigma_{r} V_{r}^{T}
$$

Where $\Sigma_{r}$ is a $r \times r$ diagonal matrix, $U_{r}$ and $V_{r}$ are matrices consisting of the first $r$ columns of $U$ and $V$, respectively. Then the influence matrix can be diagonalized by the matrices $U_{r}$ and $V_{r}$.

$$
U_{r}^{T} D V_{r}=\Sigma_{r}
$$

For $\Sigma_{r}$ is invertible, pre-multiplying the inverse of $\Sigma_{r}$ with $V_{r}$ yields a normalized diagonal matrix. Choose

$$
R=U_{r}^{T} \quad, \quad Q=V_{r} \Sigma_{r}^{-1}
$$

Project the error signals from sensor space to control space and project the control signal from control space to sensor space, i.e.,

$$
e_{u}=R e_{s},\left(e_{s} \rightarrow e_{u}\right) \text { and } e_{s}=Q e_{u},\left(u_{u} \rightarrow u_{s}\right)
$$

The diagram of AO system that uncoupled by SVD is shown as Figure. IV Therefore, AO system will uncouple and normalize the feedback path from $u_{u}$ to $e_{u}$ in Figure. IV as follows.

$$
e_{u}=-R D Q u_{u}=-U_{r}^{T} U_{r} \Sigma_{r} V_{r}^{T} V_{r} \Sigma_{r}^{-1} u_{u}=-u_{u}
$$




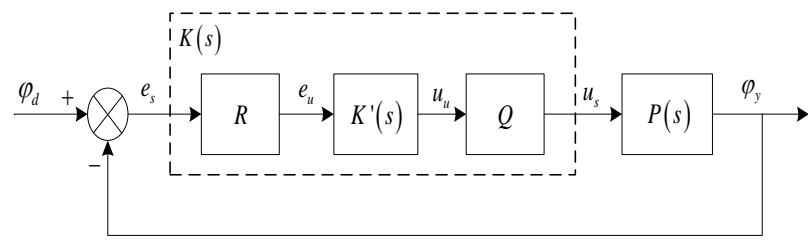
FIGURE IV. DIAGRAM OF UNCOUPLED ADAPTIVE OPTICS
SYSTEM (SVD)

\section{CONTROLLER THEORY}

This section introduces the fundamental principles of controllers that will be used for latter implementations. They are: integrator and $H_{\infty}$ synthesis.

\section{A. Integrator}

The current default controller for DM in AO system is the classical integrator which is simple and can be easily applied to engineering. The integrator can be described in discrete-time by:

$$
K(z)=\frac{g_{z}}{1-T_{a} z^{-1}}
$$

Where $z$ is the Z-transform operator, $g_{z}$ is the gain of integrator which can be adjusted according to noise and performance requirements, and $T_{a}$ is the integral coefficient which is generally set to 1 . The AO system obtains a satisfying result by opting an appropriate $g_{z}$.

\section{B. $H_{\infty}$ Synthesis}

Motivated by the desire to cope with the control challenges when dealing with the uncertainty of plant model, and to improve the robust stability and performance of AO system, we propose the use of frequency-based design technique which is based on the minimization of $H_{\infty}$ norm, i.e. $H_{\infty}$ synthesis.

Doyle et al. [25] and Zames [26] demonstrate that to compute $H_{\infty}$ solution is essentially to solve two Riccati equations in their static form (optimal estimation and optimal control problems). For a continuous-time representation, the standard configuration of $H_{\infty}$ control is shown as Figure. V:

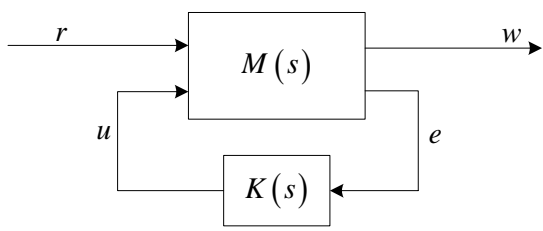

FIGURE V. STANDARD CONFIGURATIONS FOR ${ }_{\infty}$ SYNTHESIS.

The generalized plant block $M(s)$ has two inputs, the exogenous input $r$ that includes reference signal and disturbances, and the manipulated variables $u$ that make the plant work on desired. The outputs are the signals contained in vectors $w$, which will be penalized for performance and robustness as well, and $e$, which is usually the error signal in AO system, is the vector of measurements available to the controller $K(s)$. Notice that $K(s)$ is a scalar function during designing.

According to Figure. V, the plant block $M(s)$ can be represented in the Laplace domain by

$$
\left[\begin{array}{l}
w \\
e
\end{array}\right]=M(s)\left[\begin{array}{l}
r \\
u
\end{array}\right]=\left[\begin{array}{ll}
M_{11}(s) & M_{12}(s) \\
M_{21}(s) & M_{22}(s)
\end{array}\right]\left[\begin{array}{l}
r \\
u
\end{array}\right]
$$

The diagram of adaptive optics system from control perspective is depicted as Figure. II. By defining $r=\varphi_{d}+n$, control problem can be rearranged to form from the perspective of $H_{\infty}$ synthesis that is called the augmented representation shown in Figure. VI.

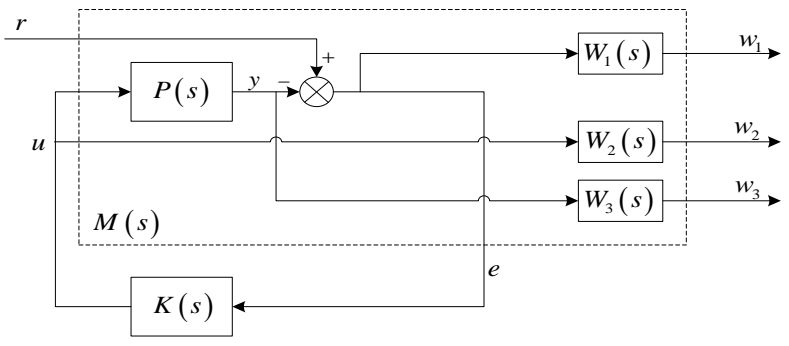

FIGURE VI. AUGMENTED CONFIGURATIONS FOR $H_{\infty}$ SYNTHESIS, WEIGHT FUNCTIONS $W_{1}(s), W_{2}(s)$ AND $W_{3}(s)$ WERE INTRODUCED TO PENALIZE ERROR, CONTROL SIGNAL AND THE NOISE, RESPECTIVELY.

Figure. VI gives the general structure of AO system from $H_{\infty}$ control point of view. The generalized plant $M(s)$ contains the plant of AO system $P(s)$ and weight functions $W_{1}(s), W_{2}(s)$ and $W_{3}(s)$. According to the general structure in Figure. VI and Equation (12), it is easy to educe the generalized plant $M(s)$, i.e.

$$
M(s)=\left[\begin{array}{ll}
M_{11}(s) & M_{12}(s) \\
M_{21}(s) & M_{22}(s)
\end{array}\right]=\left[\begin{array}{c:c}
W_{1}(s) & -W_{1}(s) P(s) \\
0 & W_{2}(s) \\
0 & W_{3}(s) P(s) \\
\hdashline I & -P(s)
\end{array}\right]_{3)}
$$

Where

$$
M_{11}(s)=\left[\begin{array}{c}
W_{1}(s) \\
0 \\
0
\end{array}\right], \quad M_{12}(s)=\left[\begin{array}{c}
-W_{1}(s) P(s) \\
W_{2}(s) \\
W_{3}(s) P(s)
\end{array}\right],
$$




$$
M_{21}(s)=I, M_{22}(s)=-P(s)
$$

And the closed-loop system from input $r$ to output $w$ in Figure. VI can be expressed by:

$$
w=F_{l}(M, K) r
$$

where $F_{l}(M, K)$ is:

$$
F_{l}(M, K)=M_{11}+M_{12} K\left(I-M_{22} K\right)^{-1} M_{21}
$$

According to the small gain theory, the sufficient condition for the stability of AO system satisfies the next inequation.

$$
\left\|F_{l}(M, K) \cdot W_{\Delta} \cdot \Delta\right\|_{\infty}<1,\|\Delta\|_{\infty} \leq 1
$$

Where $\Delta$ is unmodelled dynamic of plant, and $W_{\Delta}$ is weight function of uncertainty. The smaller of $\left\|F_{l}(M, K) \Delta\right\|_{\infty}$ is, the better of robustness will be. But equation (17) is conservative for it is a sufficient condition. Therefore, define the equation (18) as an assistant index to weigh the robustness and use the phase margin to weigh the stability.

$$
\rho=\left\|F(M, K) \cdot W_{\Delta}\right\|_{\infty}
$$

Let $S F(s), C S F(s)$ and $T F(s)$ be the sensitivity function (also known as error transfer function), control sensitivity function and complementary sensitivity function (also known as close loop transfer function or noise transfer function) respectively. They are given by:

$$
\begin{gathered}
S F(s)=\frac{e(s)}{r(s)}=\frac{1}{1+P(s) K(s)} \\
\operatorname{CSF}(s)=\frac{u(s)}{r(s)}=\frac{K(s)}{1+P(s) K(s)} \\
T F(s)=\frac{y(s)}{r(s)}=\frac{P(s) K(s)}{1+P(s) K(s)}
\end{gathered}
$$

The objective of $H_{\infty}$ synthesis is to find a stabilizing and causal linear controller $K$ that stabilizes the closed-loop operator $F_{l}(M, K)$ and enforces the norm bound $\left\|F_{l}(M, K)\right\|_{\infty}<\gamma$, in which $\gamma$ is a constant.

By defining $S F(s), \operatorname{CSF}(s)$ and $T F(s), F_{l}(M, K)$ can be deducted as Equation (22):

$$
w=F_{l}(M, K) r=\left[\begin{array}{c}
W_{1}(s) \operatorname{SF}(s) \\
W_{2}(s) \operatorname{CSF}(s) \\
W_{3}(s) T F(s)
\end{array}\right] r
$$

Then the norm bound condition becomes as Equation

$$
\left\|F_{l}(M, K)\right\|_{\infty}=\left\|\begin{array}{c}
W_{1}(s) S F(s) \\
W_{2}(s) \operatorname{CSF}(s) \\
W_{3}(s) T F(s)
\end{array}\right\|_{\infty}<\gamma
$$

It is a key process for $H_{\infty}$ control to choose the right weight functions. The weight function $W_{1}(s)$ is a low pass filter to shape the sensitivity function $S F(s)$. So the sensitivity function is a high pass filter and can minimize the error signal $e$. The weighting function $W_{2}(s)$ is a high pass filter with a crossover frequency that approximately equals to the desired closed loop bandwidth. It contributes to the robustness of the closed loop system by minimizing the control signal $u$. The weighting function $W_{3}(s)$ is a high pass filter to shape the complementary sensitivity function $T F(s)$. So the complementary sensitivity function is a low pass filter that can restrain the high frequency element of noise. An excellent controller could be designed by choosing the appropriate weighting functions.

\section{CONTROLler DESIGN}

In this section, controllers designed in uncoupled channels and the frequency response analysis in SISO loop is presented. Then the controller is analyzed in MIMO loop to simulate the real engineering.

\section{A. Integrator}

The classical integrator achieves a satisfactory performance by opting an appropriate gain $g_{z}$. It decides bandwidths and overshoots in AO system. For AO system, the open loop gain can be normalized by SVD arithmetic, but the time delay can not be normalized. We consider it is about 2-3 periods. To simplify the process of designing controller, we select the identical gain $g_{z}$ for each loop instead of designing different $g_{z}$, and provide enough phase margins to guarantee stability. For sampling period $T_{s}=0.001 \mathrm{~s}$, considering open loop gain is 1 and time delay $\tau=2 T_{s}$ as the normal model to select the $g_{z}$. Table I shows the phase margin value and Figure. VII shows the frequency responses of error transfer function and Figure. VIII shows close-loop transfer function (right) with integrator gain $\left(g_{z}=\left[\begin{array}{lllll}0.15 & 0.2 & 0.25 & 0.3 & 0.35\end{array}\right]\right)$. Table I illuminates that the phase margin decreases as the $g_{z}$ increases. Figure. VII and Figure. VIII reveals that both bandwidths and overshoots will be enhanced as the gain $g_{z}$ increases, but the capacity to restrain noise in high frequency will be limited. To guarantee stability of the 
system, the phase margin should be about $60^{\circ}$. And to obtain a good dynamic performance, the overshoot of error transfer function is made as no more than $5 \mathrm{~dB}$. Based on Table I and Figure. VII, choose the gain $g_{z}$ as 0.35, i.e. ,

$$
k_{i}(z)=\frac{0.35}{1-z^{-1}}
$$

Under this condition, the overshoot of $S F(s)$ is about $4.72 \mathrm{~dB}$ and the phase margin is about $59.343^{\circ}$.

TABLE I. PHASE MARGIN AND OVERSHOOT (SF) WITH $g_{z}=\left[\begin{array}{lllll}0.15 & 0.2 & 0.25 & 0.3 & 0.35\end{array}\right]$

\begin{tabular}{|c|c|c|c|c|c|}
\hline$g_{z}$ & 0.15 & 0.2 & 0.25 & 0.3 & 0.35 \\
\hline $\begin{array}{c}\text { Phase } \\
\text { margin }\end{array}$ & $77.0615^{\circ}$ & $72.7009^{\circ}$ & $68.2997^{\circ}$ & $63.8473^{\circ}$ & $59.343^{\circ}$ \\
\hline $\begin{array}{c}\text { Oversho } \\
\text { ot (SF) }\end{array}$ & $1.84 \mathrm{~dB}$ & $2.5 \mathrm{~dB}$ & $3.19 \mathrm{~dB}$ & $3.93 \mathrm{~dB}$ & $\begin{array}{c}4.72 \mathrm{~d} \\
\text { B }\end{array}$ \\
\hline
\end{tabular}

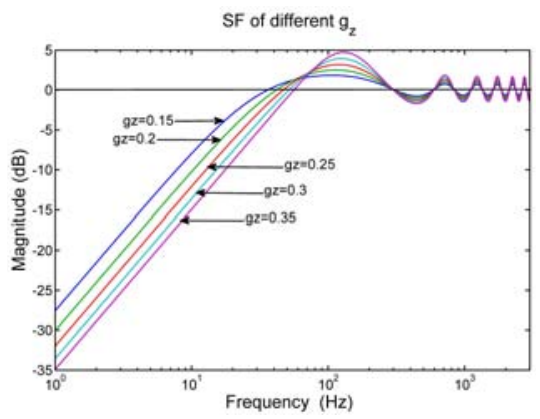

FIGURE VII. FREQUENCY RESPONSE OF ERROR TRANSFER FUNCTION FOR INTEGRATOR IN UNCOUPLED CHANNEL WITH $g_{z}=\left[\begin{array}{lllll}0.15 & 0.2 & 0.25 & 0.3 & 0.35\end{array}\right]$.

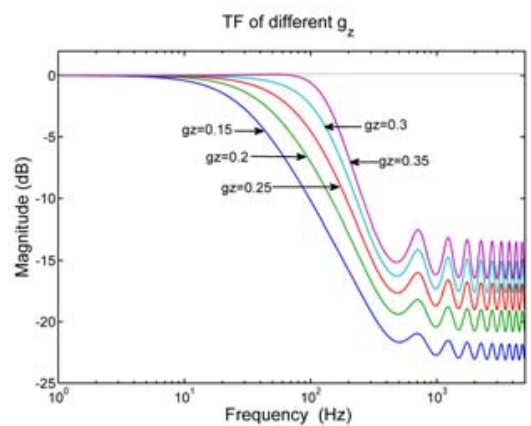

FIGURE VIII. FREQUENCY RESPONSE OF NOISE TRANSFER FUNCTION FOR INTEGRATOR IN UNCOUPLED CHANNEL WITH $g_{z}=\left[\begin{array}{lllll}0.15 & 0.2 & 0.25 & 0.3 & 0.35\end{array}\right]$.

\section{B. $H_{\infty}$ Synthesis}

Same as section 4.1, considering the time delay $2 T s \leq \tau \leq 3 T s$. Choose the normal model as the bilinear model with time delay $\tau=2 T s$ and loop gain $k=1$ (normalized by decomposition) in uncoupled channels. By repeated attempts, the weight function of uncertainty is selected as Figure. IX (red line).

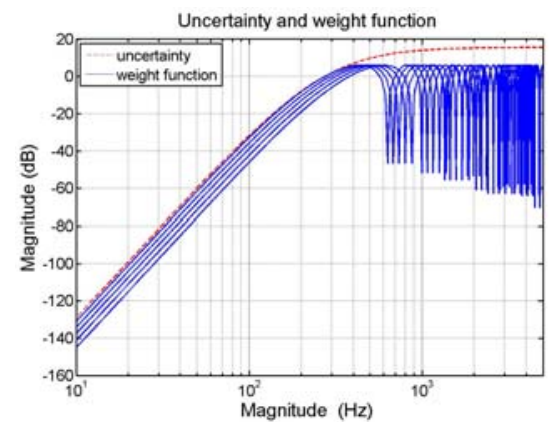

FIGURE IX. BODE PLOTS OF WEIGHT FUNCTION (RED DASHED LINE) AND UNCERTAINTY (BLUE SOLID LINE)

There is no general method to select feat weight functions to design the $H_{\infty}$ controller. Through repeated attempts, the weight functions of sensitivity function, control sensitivity function and complementary sensitivity function can be chose as equation (4.3 4.5) respectively. $W_{1}(s)$ is a low-pass filter, $W_{2}(s)$ is a high-pass filter, and $W_{3}(s)$ chooses 0.1 in all frequency to limit the noise. Figure. $\mathrm{X}$ gives the bode plots of the inverse of weight functions.

$$
\begin{gathered}
W_{1}(s)=\frac{s+1060}{2 s+15.14} \\
W_{2}(s)=\frac{s+265}{0.01429 s+530} \\
W_{3}(s)=0.1
\end{gathered}
$$

Then the controller can be solved and discretized by using a bilinear approximation as follows:

$$
k_{r}(z)=\frac{0.33 z^{5}+0.24 z^{4}-0.35 z^{3}-0.22 z^{2}+0.02 z-0.02}{z^{5}-0.53 z^{4}-1.09 z^{3}+0.14 z^{2}+0.13 z+0.35}(28)
$$

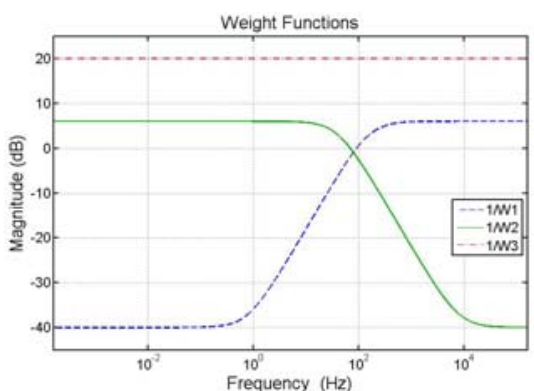

FIGURE X. BODE PLOTS OF THE INVERSES OF WEIGHT FUNCTIONS

To analyze the dynamic performance and stability of integrator and $H_{\infty}$ controller, frequency responses of two states (time delay $\tau=2 T s$ and $\tau=3 T s$ ) are simulated. Table II gives a detailed summary and Figure. XI and Figure. 
XII shows the frequency response of $S F(s)$ and $T F(s)$ in uncouple channel while $\tau=2 T s$. It shows that the dynamic performance differs slightly between integrator and $H_{\infty}$ controller. However, the phase margin is $59.343^{\circ}$ for integrator and $66.8423^{\circ}$ for $H_{\infty}$ controller (the former is less than the latter about $7.5^{\circ}$ ), indicating $H_{\infty}$ controller has greater stability than integrator. And the robustness index also displays that $H_{\infty}$ controller outweighs integrator slightly.

Table III gives a detailed summary and Figure. XIII and XIV shows the frequency responses of $S F(s)$ and $T F(s)$ in uncouple channel while $\tau=3 T s$. According to expectation, increasing the time delay will bring a negative influence on the system. For sensitivity functions $S F(s)$, the overshoots increase to $8.58 \mathrm{~dB}$ for integrator and $6.95 \mathrm{~dB}$ for $H_{\infty}$ controller, and the error restrain bandwidths are both reduced to $44 \mathrm{~Hz}$. For close-loop transfer functions, the overshoots increase to $5.43 \mathrm{~dB}$ for integrator and $2.92 \mathrm{~dB}$ for $H_{\infty}$ controller, and the $-3 \mathrm{~dB}$ bandwidths are both about $132 \mathrm{~Hz}$. The phase margins are also minished from $59.343^{\circ}$ to $38.9752^{\circ}$ for integrator and from $66.8423^{\circ}$ to $48.4035^{\circ}$ for $H_{\infty}$ controller. But the latter is greater than former about $9.4284^{\circ}$.

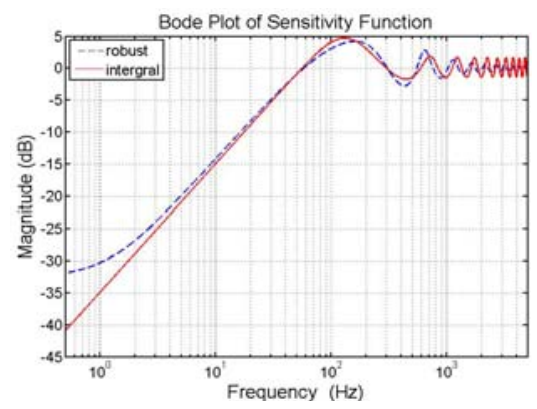

FIGURE XI. FREQUENCY RESPONSES OF SENSITIVITY FUNCTIONS FOR $\tau=2 T s$.

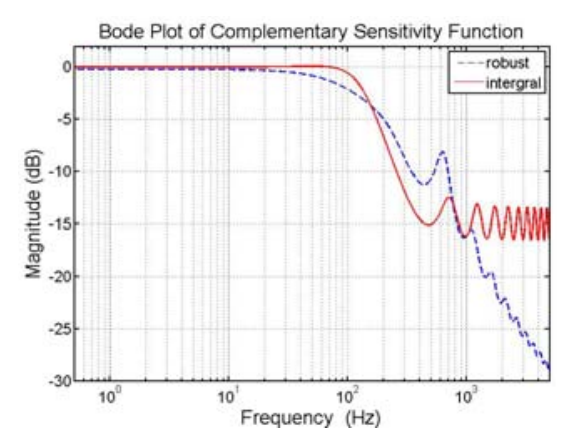

FIGURE XII. FREQUENCY RESPONSES OF COMPLEMENTARY SENSITIVITY FUNCTIONS FOR $\tau=2 T s$

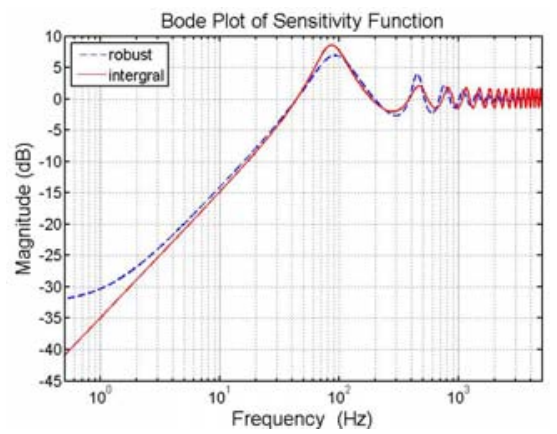

FIGURE XIII. FREQUENCY RESPONSES OF SENSITIVITY FUNCTIONS FOR $\tau=3 T s$.

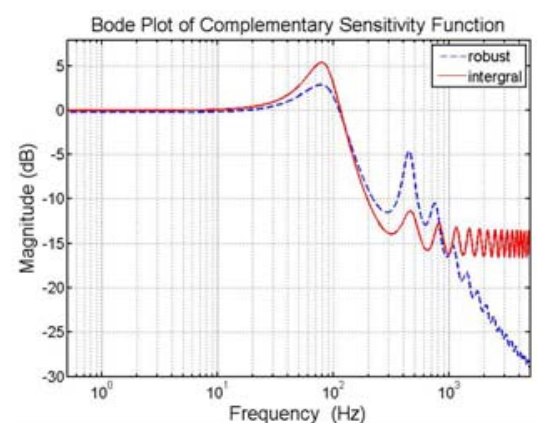

FIGURE XIV. FREQUENCY RESPONSES OF COMPLEMENTARY SENSITIVITY FUNCTIONS FOR $\tau=3 T s$.

TABLE II. SUMMARY OF THE TWO CONTROL METHODS WITH $\tau=2 T s$

\begin{tabular}{|c|c|c|c|c|c|c|}
\hline & $\begin{array}{c}\text { Oversho } \\
\text { ots of SF }\end{array}$ & $\begin{array}{c}f_{0 d B} \\
\mathbf{( S F})\end{array}$ & $f_{-3 d b}$ & $\begin{array}{c}\text { Phase } \\
\text { margin } \\
\mathbf{( T F )}\end{array}$ & $\begin{array}{c}\text { Gain } \\
\text { margi } \\
\mathbf{n}\end{array}$ & $\rho$ \\
\hline $\begin{array}{c}\text { integr } \\
\text { al }\end{array}$ & $\approx 4.72 d B$ & $\begin{array}{c}56.1 \\
\mathrm{~Hz}\end{array}$ & $\begin{array}{c}147 \mathrm{H} \\
\mathrm{z}\end{array}$ & $59.343^{\circ}$ & 2.599 & $\begin{array}{c}0.904 \\
6\end{array}$ \\
\hline $\begin{array}{c}H_{\infty} \\
\text { robust }\end{array}$ & $\approx 4.22 \mathrm{~dB}$ & $\begin{array}{c}57.8 \\
\mathrm{~Hz}\end{array}$ & $\begin{array}{c}135 \mathrm{H} \\
\mathrm{z}\end{array}$ & $66.8423^{\circ}$ & $\begin{array}{c}2.657 \\
5\end{array}$ & $\begin{array}{c}0.876 \\
7\end{array}$ \\
\hline
\end{tabular}

TABLE III. SUMMARY OF THE TWO CONTROL METHODS WITH $\tau=3 T s$

\begin{tabular}{|c|c|c|c|c|c|c|}
\hline & $\begin{array}{c}\text { Oversho } \\
\text { ots of SF }\end{array}$ & $\begin{array}{c}\text { Oversho } \\
\text { ots of } \\
\text { CSF }\end{array}$ & $f_{0 d B}$ & $f_{-3 d b}$ & $\begin{array}{c}\text { Phase } \\
\text { margin }\end{array}$ & $\begin{array}{c}\text { Gain } \\
\text { margi } \\
\mathbf{n}\end{array}$ \\
\hline $\begin{array}{c}\text { integr } \\
\text { al }\end{array}$ & $\approx 8.58 d B$ & $\approx 5.43 d B$ & $\begin{array}{c}44 \mathrm{H} \\
\mathrm{z}\end{array}$ & $\begin{array}{c}132 \\
\mathrm{~Hz}\end{array}$ & $38.9752^{\circ}$ & $\begin{array}{c}1.703 \\
1\end{array}$ \\
\hline $\begin{array}{c}H_{\infty} \\
\text { robus } \\
\mathbf{t}\end{array}$ & $\approx 6.95 d B$ & $\approx 2.92 d B$ & $\begin{array}{c}44 \mathrm{H} \\
\mathrm{z}\end{array}$ & $\begin{array}{c}133 \\
\mathrm{~Hz}\end{array}$ & $48.4035^{\circ}$ & $\begin{array}{c}1.931 \\
1\end{array}$ \\
\hline
\end{tabular}

\section{V.NUMERICAL DESIGN FOR ADAPTIVE OPTICS SYSTEMS}

Last section indicates that $H_{\infty}$ controller can improve the stability of system. And we expect that it will maintain the performance of AO system. Therefore, we give a computer simulation to test the correction ability of integrator and $H_{\infty}$ controller in this section.

\section{A. Atmospheric Turbulence Data}

Atmospheric turbulence data which satisfy the 
Kolmogorov statistics characteristics can be generated with the matlab toolbox (Object-Oriented Matlab Adaptive Optics, OOMAO), which is a special toolbox for AO system simulation. According to Kolmogorov frozen hypothesis, the phase structure function of Kolmogorov turbulence can be expressed as [23]:

$$
D(r)=\left\langle[\varphi(x)-\varphi(x+r)]^{2}\right\rangle=6.88\left(\frac{r}{r_{0}}\right)^{5 / 3}
$$

Where, $\varphi(x)$ denotes the wavefront phase at point $x$, $r$ denotes the distance, $r_{0}$ is the Fried parameter, and $\langle\cdot\rangle$ is mean operator.

A two-layer turbulence model is used, and parameters are depicted as Table IV. The wind direction is defined as: the horizontal right is positive direction of $x$-label and the vertical upward is positive direction of $y$-label. In this condition, 500 frames (0.5 second) turbulences are generated with OOMAO.

The Phase structure function is depicted as Figure. XIX. The red-solid line is theoretical value and black-dashed line is simulated value. It can be found that the simulate turbulences are tally with the theoretical turbulence. Figure. $\mathrm{XX}$ shows the root mean square (RMS) values and peak-to valley (PV) values of turbulence. The mean of RMS is $2.7571 \mathrm{rad}$, and the mean of $\mathrm{PV}$ is $13.5383 \mathrm{rad}$.

TABLE IV. THE PARAMETERS OF SIMULATION FOR TURBULENCE

\begin{tabular}{|c|c|}
\hline Telescope Diameter (D) & $1.2 \mathrm{~m}$ \\
\hline Field of View & $1.5 \mathrm{arcmin}$ \\
\hline Two layers & {$[05000] \mathrm{m}$} \\
\hline Wind speed & {$[520] \mathrm{m} / \mathrm{s}$} \\
\hline Wind direction & {$[090]$ degree } \\
\hline$r_{0}$ & $20 \mathrm{~cm}$ \\
\hline$L_{0}$ & $30 \mathrm{~cm}$ \\
\hline Wavelength & $640 \mathrm{~nm}$ \\
\hline Sampling frequency & $1000 \mathrm{~Hz}$ \\
\hline Resolution & $300 \times 300$ \\
\hline
\end{tabular}

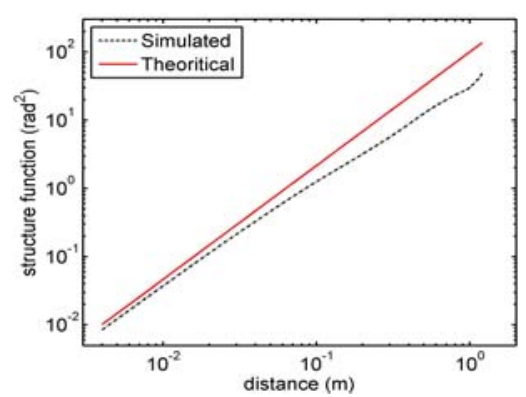

FIGURE XV. PHASE STRUCTURE FUNCTION, RED-SOLID LINE IS THEORETICAL VALUE AND BLACK-DASHED LINE IS SIMULATED VALUE.

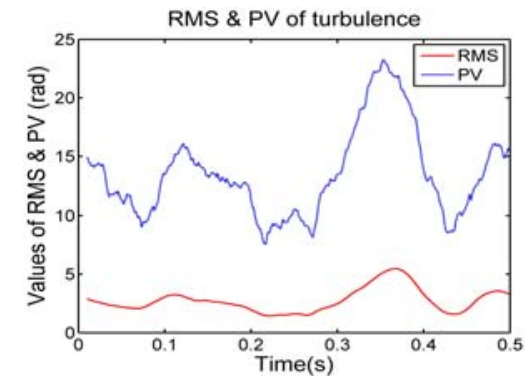

FIGURE XVI. RMS AND PV VALUE OF TURBULENCE (WITHOUT NOISE), RED-SOLID LINE IS RMS VALUE (RMS MEAN is 2.7571 RAD) AND BLUE-DASHED LINE IS PV VALUE (PV MEAN IS 13.5383 RAD).

\section{B. Time Delay $\tau=2 T$ s}

Considering the state for time delay $\tau=2 T$ s, we give a new definition about the correction efficiency as follows:

$$
\eta=\frac{R M S_{\text {residual }}}{R M S_{\text {turbulence }}}
$$

Where $R M S_{\text {residual }}$ denotes the RMS value of residual, and $R M S_{\text {turbulence }}$ denotes the RMS value of turbulence. The smaller the $\eta$ value is, the better the correct efficiency will be. Actually, noise is unavoidable in AO system and can be supposed as gauss white noise. We define the signal to noise ratio as equation (31).

$$
S N R=\frac{R M S_{\text {signal }}}{R M S_{\text {noise }}}
$$

Where, $R M S_{\text {signal }}$ is the RMS of signal and $R M S_{\text {noise }}$ is the RMS of noise. Here, choose the SNR is 10 .

Figure. XXI shows the RMS value of residual and Figure. XXII shows $\eta$ value of AO system. Figure. XXIII shows PV value of residual and Figure XXIV shows control voltage. And Table $\mathrm{V}$ gives a summary of correction while $\tau=2 T s$. For RMS mean, integrator is $0.5484 \mathrm{rad}$ and $H_{\infty}$ controller is $0.5611 \mathrm{rad}$. For correction efficiency $\eta$ value, integrator is 0.2225 and $H_{\infty}$ controller is 0.2267 . And For residual PV, integrator is $6.7203 \mathrm{rad}$ and $H_{\infty}$ controller is $6.4384 \mathrm{rad}$. The residual RMS, residual PV and $\eta$ value explain that the two methods are differing slightly for correct ability from each other. But for mean of control voltage PV and RMS of control voltage PV, $H_{\infty}$ control (112.9533 for PV mean and 43.3459 for RMS of control voltage) is reduced about $6.51 \%$ and $5.12 \%$ respectively relative to integrator (119.0448 for PV mean and 46.3649 for RMS of control voltage). This illuminates that $H_{\infty}$ control is more stable than integrator. 
TABLE V. SUMMARY OF THE PERFORMANCE WITH $\tau=2 T \mathrm{~s}$

\begin{tabular}{|c|c|c|c|c|c|}
\hline & $\begin{array}{c}\text { RMS } \\
\text { mean } \\
\text { (rad) }\end{array}$ & $\begin{array}{c}\text { PV } \\
\text { mean } \\
\text { (rad) }\end{array}$ & $\begin{array}{c}\eta \\
\text { mean }\end{array}$ & $\begin{array}{c}\text { RMS of } \\
\text { Voltage } \\
\text { PV (V) }\end{array}$ & $\begin{array}{c}\text { Mean of } \\
\text { Voltage } \\
\text { PV (V) }\end{array}$ \\
\hline integrator & 0.5484 & 6.7203 & 0.2225 & 46.3649 & 119.0448 \\
\hline$H_{\infty}$ control & 0.5611 & 6.4384 & 0.2267 & 43.3459 & 112.9533 \\
\hline
\end{tabular}

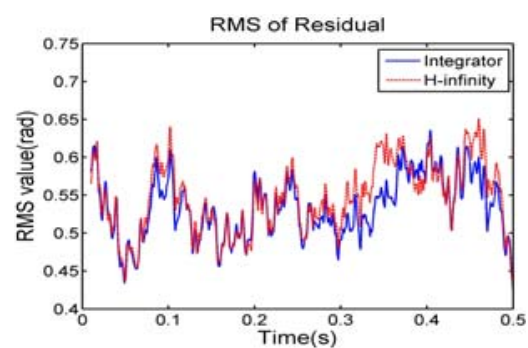

FIGURE XVII. RMS VALUE OF RESIDUAL $\tau=2 T S$ (BLUE-SOLID LINE IS FOR INTEGRATOR AND RED-DASHED LINE IS FOR ROBUST CONTROL)

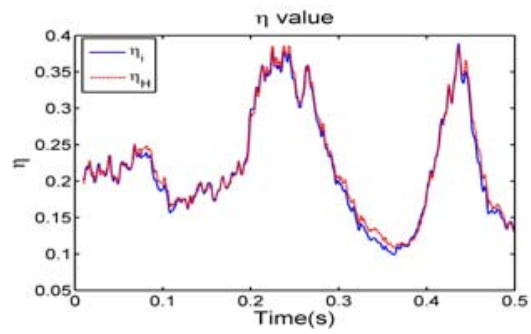

FIGURE XVIII. EFFICIENCY OF CORRECTION $\tau=2 T \mathrm{~s}$ (BLUE-SOLID LINE IS FOR INTEGRATOR AND RED-DASHED LINE IS FOR ROBUST CONTROL).

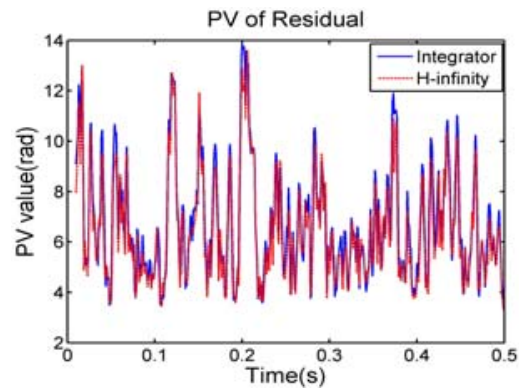

FIGURE XIX. PV OF RESIDUAL $\tau=2 T s$.

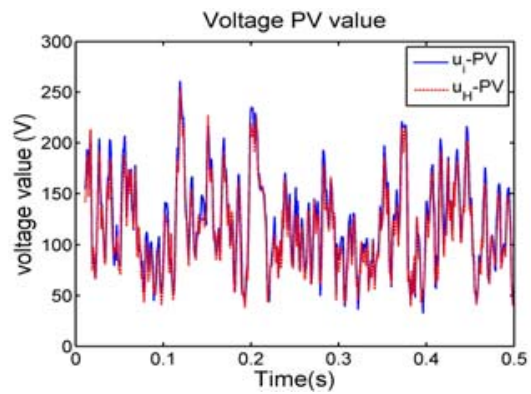

FIGURE XX. PV OF CONTROL VOLTAGE $\tau=2 T s$.

\section{Time delay $\tau=3 T$ s}

Consider the state for time delay $\tau=3 T s$, the RMS value is shown in Figure. XXV and $\eta$ value is shown in Figure. XXVI. The PV value of residual is shown in Figure. XXVII and control voltage is shown in Figure. XXVIII. And a summary of the performance is given in Table VI. By comparing the results of $\tau=2 T s$ and $\tau=3 T s$, it is easy to know that time delay will exert negative influence on $\mathrm{AO}$ system.

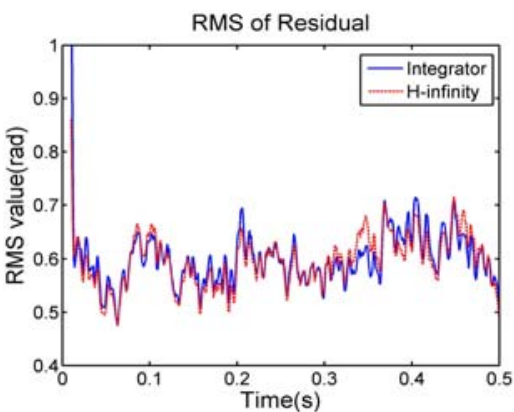

FIGURE XXI. RMS VALUE OF RESIDUAL $\tau=3 T s$ (BLUE-SOLID LINE IS FOR INTEGRATOR AND RED-DASHED LINE IS FOR ROBUST CONTROL).

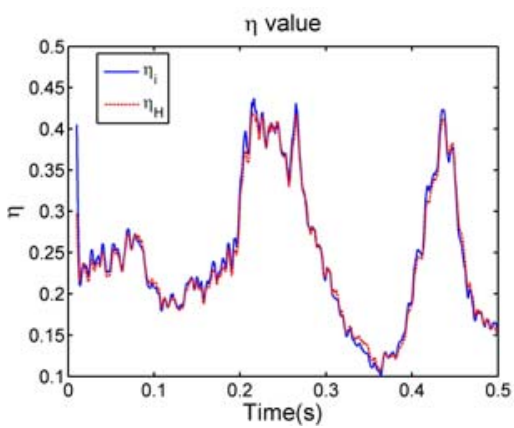

FIGURE XXII. EFFICIENCY OF CORRECTION $\tau=3 T s$ (BLUE-SOLID LINE IS FOR INTEGRATOR AND RED-DASHED LINE IS FOR ROBUST CONTROL).

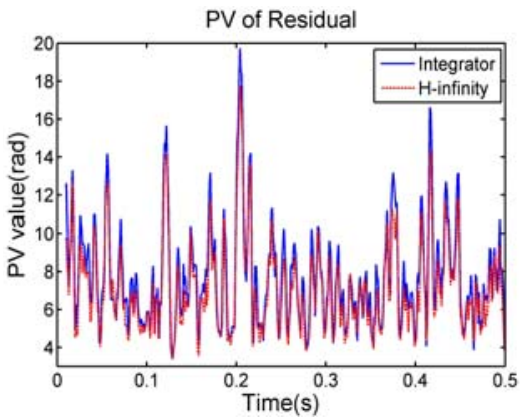

FIGURE XXIII. PV VALUE OF RESIDUAL ( $\tau=3 T s$ ). 


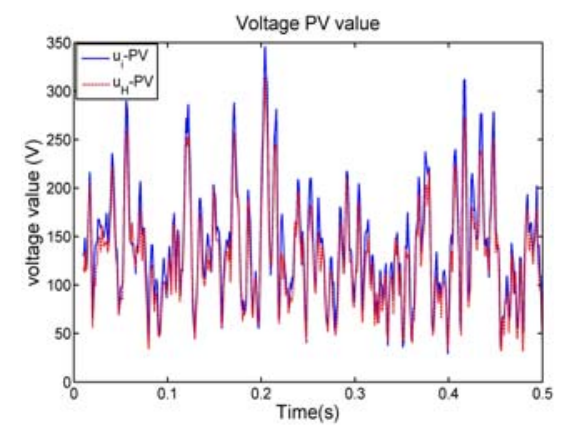

FIGURE XXIV. PV OF CONTROL VOLTAGE ( $\tau=3 T s$ ).

TABLE VI. SUMMARY OF THE PERFORMANCE WITH $\tau=3 T s$

\begin{tabular}{|c|c|c|c|c|c|}
\hline & $\begin{array}{c}\text { RMS } \\
\text { mean } \\
\text { (rad) }\end{array}$ & $\begin{array}{c}\text { PV } \\
\text { mean } \\
\text { (rad) }\end{array}$ & $\begin{array}{c}\eta \\
\text { mean }\end{array}$ & $\begin{array}{c}\text { RMS of } \\
\text { Voltage } \\
\text { PV (V) }\end{array}$ & $\begin{array}{c}\text { Mean of } \\
\text { Voltage } \\
\text { PV (V) }\end{array}$ \\
\hline integrator & 0.6219 & 7.7092 & 0.2523 & 57.2290 & 136.5921 \\
\hline$H_{\infty}$ control & 0.6166 & 7.1093 & 0.2494 & 51.7907 & 124.0501 \\
\hline
\end{tabular}

For time delay $\tau=3 T s$, integrator has a slightly better correction than $H_{\infty}$ controller. However, when $\tau=3 T$, $H_{\infty}$ controller becomes better slightly. Table VI shows the difference clearly while $\tau=3 T s$. The RMS mean of residual is 0.6219 rad for the integrator and 0.6166 rad for $H_{\infty}$ controller. And in terms of the mean of $\eta$ value, the integrator is 0.2523 and $H_{\infty}$ controller is 0.2494 . The PV mean of residual is 7.7092 rad for the integrator and 7.1093 rad for the $H_{\infty}$ controller. The latter recedes about $4.43 \%$ comparing to integrator. $H_{\infty}$ controller will not cost the correct capacity to improve the stability. For the RMS of voltage PV, the integrator is $57.2290 \mathrm{~V}$ and $H_{\infty}$ controller is $51.7907 \mathrm{~V}$, the latter recedes about $9.5 \%$ comparing to integrator. And for the mean of voltage of PV, the integrator is $136.5921 \mathrm{~V}$ and $H_{\infty}$ controller is $124.0501 \mathrm{~V}$, the latter recedes about $9.18 \%$ comparing to integrator. These two parameters illuminate that for the same turbulence, $H_{\infty}$ control can calculate a low control voltage to achieve the same performance as integrator. That is to say, the stability of $\mathrm{AO}$ system is enhanced.

\section{CONCLUSION}

To improve the robust stability of AO system, $H_{\infty}$ synthesis has been introduced to design the controller. This paper first makes the MIMO model of AO system. In order to simplify the design of controller, we decompose the coupled loop and design SISO controller in uncoupled channel. Then we analyze the frequency responses in uncoupled channels with $H_{\infty}$ synthesis and integrator. Via comparing integrator with $H_{\infty}$ synthesis, it is concluded that the latter improves phase margin about $7.4993^{\circ}$ while $\tau=2 T s$ and about $9.4283^{\circ}$ while $\tau=3 T s$. The assistant index $\rho$ also shows that the latter has better robustness for it reduces about 0.0279 . Lastly we simulate the turbulence correction of AO system to test the performance of two controllers. Actually, $H_{\infty}$ synthesis and integrator have nearly the same correct ability while time delay $\tau=2 T s$ and time delay $\tau=3 T s$. But by comparing the RMS and mean of control voltage PV between the two methods, it implies that $H_{\infty}$ synthesis achieves the same performance as integrator with a lower control voltage when turbulence is the same. Therefore, it is concluded that $H_{\infty}$ control improves stability without sacrificing the performance of AO system.

\section{ACKNOWLEDGMENTS}

This work has been supported by the Key Laboratory on Adaptive Optics, Institute of Optics and Electronics, Chinese Academy of Sciences, and University of Electronic Science and Technology of China.

\section{REFERENCE}

[1]. N. Denis, D. Looze, J. Huang, and D. Castanon, “ $H_{\infty}$ control design for an adaptive optics system,” Kybernetika 35(1), 69-81(1999).

[2]. D. W. Miller, and S. C. O. Grocott, "Robust control of the Multiple Mirror Telescope adaptive secondary mirror,” Opt. Eng. 38(8), 1276-1287 (1999).

[3]. B. W. Frazier, R. K. Tyson, and M. S. J. Roche, "Theory and operation of a robust controller for a compact adaptive optics system," Opt. Eng. 43(12), 2912-2920 (2004).

[4]. P. Piatrou, and L. Gilles, "Robustness study of the pseudo open-loop controller for multiconjugate adaptive optics,” Appl. Opt. 44(6), 1003-1010 (2005).

[5]. R. N. Paschall, and D. J. Anderson, "Linear quadratic Gaussian control of a deformable mirror adaptive optics system with time-delayed measurements,” Appl. Opt. 32(31), 6347 -6358 (1993).

[6]. D. P. Looze, "Minimum variance control structure for adaptive optics systems,” J. Opt. Soc. Am. A. 23(3), 603-612(2006).

[7]. J. P. Folcher, and M. Carbillet, "Numerical atmospheric turbulence models and LQG control for adaptive optics system," Proc. SPIE 8172, Optical Complex Systems: OCS11, 81721C (2011).

[8]. C. Correia, H. F. Raynaud, C. Kulcsar, and J. M. Conam, "Minimum-variance control for astronomical adaptive optics with resonant deformable mirrors," European Journal of Control 3, 222-236 (2011).

[9]. Yu-tai Liu, and S. Gibon, "Adaptive control in adaptive optics for directed-energy systems,” Optical Engineering 46(4), 046601(2007).

[10]. G. Agapito, S. Baldi, G. Battistelli, D. Mari, E. Mosca, and A. Riccardi, " Automatic tuning of the internal position control of a adaptive secondary mirror,” European Journal of Control (3), 273 -289 (2011).

[11]. R. Muradore, L. Perrazzi, R. Clare, and E. Fedrigo, “ An application of adaptive techniques to vibration rejection in adaptive optics systems," Control Engineering Practice (32), 87-95 (2014).

[12]. M. Manetti, M. Morandini, and P. Mantegazza, "Self-tuning shape control of massively actuated adaptive mirrors," IEEE transactions on control systems technology 22(3), 838-852 (2014).

[13]. L. Poyneer, and J. P. Veran, "Predictive wavefront control for adaptive optics with arbitrary control loop delays,” J. OPT. Soc. Am. A 25(7), 1486-1496 (2008).

[14]. Li Xinyang, and Luo Qi, and Yan Zhaojun, "Prediction control method to improve the dynamic performance of a close-loop adaptive optics system," Proc. SPIE 9909, Adaptive Optics Systems V, 99094W (2016).

[15]. P.C. Mcguire, T. A. Rhoadarmer, H. Coy, J. R. P. Angel, M. L. Hart, "Linear zonal atmospheric prediction for adaptive optics", Proc. SPIE 4007, Adaptive Optical Systems Technology, 4007: 682-691 (2000).

[16]. Yan Zhaojun, Li Xinyang, and Rao Changhui, "Multi-channel Adaptive control algorithm for closed-loop adaptive optics system," Acta Optica Sinica 33(3), 0301002 (2013).

[17]. A.Guesalaga, D. Guzman, R. Myers, R. Sharples, T. Morris, C. 
Saunter, and A. Basden, "Synthesis of approximate zonal controllers for MEMS DMs,” Proc. SPIE 6888, MEMS Adaptive Optics II, $68880 \mathrm{~K}(2008)$.

[18]. A.Guesalaga, D. Guzman, R. Myers, T. Morris, N. Dipper, and A. Basden, "Control of deformable mirrors in MOAO using $H_{\infty}$ optimization,” Proc. SPIE 7439, Astrinimical and Space Optical Systems, 74390N(2009).

[19]. A. Guesalaga, B. Neichel, F. Rigaut, J. Osborn, and D. Guzman, "Comparison of vibration mitigation controllers for adaptive optics systems," Appl. Opt. 51(19), 4520-4535(2012).

[20]. A. Guesalaga, B. Neichel, J. O’Neal, and D. Guzman, "Mitigation of vibrations in adaptive optics by minimization of closed-loop residuals," Opt. Express 21(9), 10676-10696(2013).

[21]. Dingan Song, Xinyang Li, and Zhenming Peng, "Mixed sensitivity H-infinity control of an adaptive optics system”, Opt. Eng, 55(9), 094106 (2016).

[22]. Yan Haixing, Zhang Deliang, and Li Shushan, "Numerical simulation of an adaptive optics system: Direct wavefront gradient control method", Acta optica sinica, 17(6), 758-765 (1997).

[23]. J. W. Hardy, "Adaptive optics for astronomical telescopes," Oxford University press, 90-92 (1998).

[24]. M. Nagashima, and B. N. Agrawal, "Active control of adaptive optics system in a large segmented mirror Telescope," International Journal of Systems Science 45 (12), 1-17 (2012).

[25]. J. C. Doyle, K. Glover, P. P. Khargonekar, and B. A. Francis, "State-space solutions to standard $\mathrm{H}_{2}$ and $\mathrm{H}_{\infty}$ control problems," IEEE Trans. Automat. Contr. 34(8), 831-847 (1989).

[26]. G. Zames, "Feedback and optical sensitivity: model reference transformations, multiplicative seminorms, and approximate inverses,” IEEE Trans. Automat. Contr. 26(2), 301-320 (1981). 